\title{
Urinary albumin excretion in patients with systemic lupus erythematosus without renal disease
}

\author{
Enrique Batlle-Gualda, Ana Carro Martínez, Rocio Alfayate Guerra, Eliseo Pascual
}

Department of Rheumatology and

University of Alicante, Hospital General Universitario de Alicante, Alicante, Spain

E Batlle-Gualda

A C Martínez

E Pascual

Department of Clinical Analysis, Hospital General Universitario de Alicante, Alicante, Spain

R A Guerra

Correspondence to: Dr E Batlle-Gualda, Sección de Reumatología, Hospital General Universitario de Alicante, Calle Maestro Alonso, 109 03010, Alicante, Spain.

Accepted for publication 26 March 1997

\begin{abstract}
Objectives-To investigate the prevalence of microalbuminuria, urinary albumin excretion (UAE) between $20-200 \mu \mathrm{g} / \mathrm{min}$, in systemic lupus erythematosus (SLE) patients without clinical renal disease, and to discover if this could predict the development of renal disease.

Methods-This study made six monthly measurements of UAE, creatinine clearance, serological and clinical data in 22 ambulatory women patients with SLE, without clinical renal disease, hypertension, diabetes or heart failure. The patients were followed up for a period of 18 months (four measurements). Age and sex matched healthy controls were used as a comparative group. UAE was measured by nephelometry in three timed overnight urine samples at each visit.

Results-There were no significant differences in the creatinine clearance between the control group and the SLE patients. Creatinine clearance did not show significant changes throughout the study period. SLE patients had wide variations in the UAE rate compared with healthy controls. In five patients $(5$ of $22 ; 23 \%)$, on occasions, there was mild, transient increase in UAE reaching the level of microalbuminuria. During follow up, one patient with basal $(4.67 \mu \mathrm{g} / \mathrm{min})$ and six month (4.73 $\mu \mathrm{g} / \mathrm{min})$ normal UAE rate, was admitted with a nephrotic syndrome confirmed on biopsy examination to be proliferative lupus nephritis. Six months after beginning treatment with prednisone and cyclophosphamide her UAE rate returned to normal values $(4.65$ $\mu \mathrm{g} / \mathrm{min})$.
\end{abstract}

Conclusion-SLE patients without clinical renal disease may have microalbuminuria, although this does not seem to warrant any specific action.

(Ann Rheum Dis 1997;56:386-389)

Urinary albumin excretion (UAE) is a more sensitive indicator than urinary total protein for the early detection of renal involvement in some chronic diseases such as diabetes mellitus and hypertension. ${ }^{1}$ Microalbuminuria has been defined as a UAE of $20-200 \mu \mathrm{g} / \mathrm{min}(30-300$ $\mathrm{mg} / 24 \mathrm{~h}$ ), which is undetectable by routine laboratory test such as dipsticks (Albustix). ${ }^{2} \mathrm{At}$ an excretion rate of about $200 \mu \mathrm{g} / \mathrm{min}$ (150-200 mg/l) conventional semiquantitative strips give a positive result for albuminuria. It has been shown that microalbuminuria is a prognostic factor of clinical nephropathy in diabetes mellitus, ${ }^{3}$ and has been associated with essential hypertension, ${ }^{4}$ and increased cardiovascular morbidity and mortality in diabetic and non-diabetic patients. ${ }^{56}$ Although poorly understood, endothelial dysfunction has been implicated in the pathogenic mechanism, and microalbuminuria may be a marker of susceptibility to increased permeability of the vascular wall.

On the other hand, microalbuminuria has also been reported in some rheumatic diseases such as rheumatoid arthritis ${ }^{89}$ and systemic sclerosis, ${ }^{10}$ and has been associated with limited joint mobility in type I diabetes mellitus, ${ }^{11}$ but until now few data exist about systemic lupus erythematosus (SLE). ${ }^{12-15}$ Several cross sectional studies made in SLE patients without clinical renal disease have shown that the UAE is frequently increased, ${ }^{1415}$ and mesangial nephropathy has been considered responsible for this, ${ }^{13}$ although other authors did not find these abnormalities. ${ }^{12}$ Whether this raised UAE may predict the development of severe renal disease in lupus patients is unknown. With the aim of better defining this issue, we have prospectively measured the UAE, every six months, in 22 SLE patients without clinical renal disease during a period of 18 months (four measurements), and compared their results with an age and sex matched healthy population as a control group.

\section{Methods}

PATIENTS

All the patients diagnosed as having SLE followed up in an outpatient clinic of rheumatology, and with at least four of the American Rheumatism Association (ARA) 1982 revised criteria for the classification of SLE $^{16}$ were included in this study, if they fulfilled the following criteria: blood glucose $<110 \mathrm{mg} / \mathrm{dl}$, blood pressure $<160 / 80 \mathrm{~mm} \mathrm{Hg}$, normal renal function, (serum creatinine $<1.3 \mathrm{mg} / \mathrm{dl}(<115$ $\mathrm{mol} / \mathrm{l}$ ) and creatinine clearance $>80 \mathrm{ml} / \mathrm{min}$ and $1.73 \mathrm{~m}^{2}$ ), and absence of abnormal proteinuria measured by the Albustix test. Patients with a history of hypertension, diabetes mellitus, renal disease or congestive heart failure were excluded. A group of age and sex matched controls were recruited from healthy hospital workers.

All the patients had a medical history taken and complete physical examination at the first visit. Thereafter patients were evaluated after six, 12, and 18 months. At each visit, a clinical 
assessment and laboratory test profile according to a previously approved protocol was obtained. Disease activity was scored according to the system used by Yeung et al. ${ }^{17}$ Blood pressure was measured with a standard mercury sphygmomanometer in both arms with the patient seated and according to previously described recommendations. ${ }^{18}$ The following laboratory tests were also performed at each visit: a complete blood count including measurement of haemoglobin, white cell differential count, and platelet count; Westergren erythrocyte sedimentation rate; serum chemistry profile; complement components (C3, C4 by immunonephelometry), antinuclear antibodies by indirect immunofluorescence on human cell line HEp-2 and ds-DNA antibodies on Crithidia lucilae as substrate; and complete urine analysis. The urine was cultured when leucocytes were found in the urine sediment examination. Infected urine samples $\left(>10^{8}\right.$ organisms $\left./ 1\right)$ were rejected. At each visit (baseline, six, 12, and 18 months) the UAE of SLE patients was measured. The measurements were made on three, eight hour overnight urine samples collected on three consecutive days, to reduce the effect of physical activity, and the mean of the three determinations was taken as the value of UAE for each visit. All the urine samples were collected avoiding menstrual periods or any intercurrent acute illnesses, and all the patients were encouraged to avoid intense exercise before urinary determinations. The samples for the controls were collected according to a similar protocol, on three, eight hour overnight urine samples, but only measured at baseline without determinations at six, 12 or 18 months. The UAE was measured by nephelometry (MA test, Beckman, Ireland) with a limit of detection of $0.2 \mathrm{mg} / \mathrm{dl}$, intra-assay variation of $\leq 2.5 \%$, and an interassay variation of $\leq 3.5 \%$. An $\mathrm{UAE}<20 \mu \mathrm{g} / \mathrm{min}$ was considered normal. Urinary and serum creatinine was measured by standard colorimetric methods. Creatinine clearance for each subject and visit was expressed as the average of the values obtained from the three urine samples collected for this particular visit. The study was carried out on outpatients.

\section{STATISTICS}

A descriptive analysis was done for all the variables. The data are expressed as mean with standard deviation (SD) for the variables normally distributed, or medians with interquartile range for the variables not normally distributed. A two tailed unpaired Student's $t$ test or Mann-Whitney U test was used to test differences between SLE patients and controls. The Friedman two way analysis of variance was used for repeated measures analysis. $p$ Values greater than 0.05 were considered non-significant. To assess the variability of the UAE in the three daily samples that compose each measurement, the coefficient of variation between them, taken in pairs, was calculated.

\section{Results}

Twenty two women of a total of 63 SLE patients were included in the study. They had a mean (SD) age of 39.1 (12.5) years (range: $23-$ 68 ) and disease duration (SD) of 7.8 (5.0) years (range: 1-21). The control group included 19 women with a mean (SD) age of 42.1 (13.3) years (range: $22-73)(\mathrm{p}=\mathrm{NS}$ ). Table 1 shows the clinical findings, laboratory data, and treatment received during the study. Twelve patients (12 of $22 ; 55 \%$ ) had had antidsDNA antibodies before starting the study. There were no significant differences in the creatinine clearance, mean (SD) (range), between the control group 122.8 (28.1) $\mathrm{ml} / \mathrm{min}$ (84-187) and the SLE patients, at baseline, six, 12, and 18 months of follow up, $130.6(55.4)(80-290)(\mathrm{p}=0.57), 127.6(44.3)$ $(81-264)(\mathrm{p}=0.68), 130.7(53.3)(81-287)(\mathrm{p}$ $=0.59), 121.4(26.2)(86-173)(\mathrm{p}=0.88)$, respectively. On the other hand, creatinine clearance in the SLE patients did not show significant changes throughout the study period (Friedman two way analysis of variance, $p=0.74$ ).

Table 1 Clinical features, serological findings, and treatment of 22 SLE patients, without clinical renal disease, during the study period

\begin{tabular}{|c|c|c|c|c|}
\hline & Basal $(n=22) n,(\%)$ & 6 month $(n=22) n,(\%)$ & 12 month $(n=16) * n,(\%)$ & 18 month $(n=20) n,(\%)$ \\
\hline \multicolumn{5}{|l|}{ Clinical } \\
\hline Arthritis & $9(41)$ & $5(23)$ & $3(19)$ & $1(5)$ \\
\hline Malar rash & $1(4.5)$ & $3(14)$ & $1(6)$ & $4(20)$ \\
\hline Raynaud & $9(41)$ & $6(27)$ & $4(25)$ & $3(15)$ \\
\hline Pleuritis & $1(4.5)$ & $1(4.5)$ & $0(0)$ & $0(0)$ \\
\hline Pericarditis & $1(4.5)$ & $0(0)$ & $1(6)$ & $0(0)$ \\
\hline \multicolumn{5}{|l|}{ Serological } \\
\hline Lymphopenia† & $7(32)$ & $8(36)$ & $6(38)$ & $8(40)$ \\
\hline ANA & $21(95)$ & $20(91)$ & $16(100)$ & $17(85)$ \\
\hline Anti-dsDNA & $5(23)$ & $5(23)$ & $4(25)$ & $3(15)$ \\
\hline $\mathrm{C} 3 \ddagger$ & $9(41)$ & $9(41)$ & $6(38)$ & $6(30)$ \\
\hline $\mathrm{C} 4 \ddagger$ & $8(36)$ & $10(45)$ & $4(25)$ & $7(35)$ \\
\hline \multicolumn{5}{|l|}{ Treatment } \\
\hline NSAID & $9(41)$ & $8(36)$ & $7(44)$ & $6(30)$ \\
\hline Prednisone & $12(55)$ & $12(55)$ & $7(44)$ & $9(45)$ \\
\hline (mean; range) & $(9.5 ; 2-20)$ & $(9.3 ; 2-40)$ & $(17 ; 2.5-45)$ & $(11 ; 2.5-20)$ \\
\hline Antimalarial agents & $15(68)$ & $13(59)$ & $9(56)$ & $12(60)$ \\
\hline Methotrexate & $0(0)$ & $1(4.5)$ & $1(6)$ & $0(0)$ \\
\hline Azathioprine & $0(0)$ & $0(0)$ & $0(0)$ & $1(5)$ \\
\hline Cyclophosphamide & $0(0)$ & $0(0)$ & $0(0)$ & $1(5)$ \\
\hline
\end{tabular}

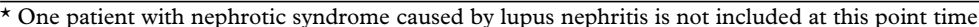

† Lymphopenia $<1500 / \mathrm{mm}^{3} ; \ddagger \mathrm{C} 3<83 \mathrm{mg} / \mathrm{dl} ; \ddagger \mathrm{C} 4<15 \mathrm{mg} / \mathrm{dl}$. 
The UAE, median (interquartile range), in the control group was 5.16 (3.33-7.67) $\mu \mathrm{g} / \mathrm{min}$. The UAE, median (interquartile range), in the SLE patients at baseline, six, 12, and 18 months were $5.40(3.87-7.60) \mu \mathrm{g} / \mathrm{min}$ $(\mathrm{p}=0.51), 6.20(4.73-7.96) \mu \mathrm{g} / \mathrm{min}(\mathrm{p}=$ $0.29), 9.38(4.83-13.46) \mu \mathrm{g} / \mathrm{min}(\mathrm{p}=0.03)$, and $8.86(4.67-13.72) \mu \mathrm{g} / \mathrm{min}(\mathrm{p}=0.03)$, respectively. Although there were no differences $(p>0.05)$ in the UAE between the SLE patients and the control group at baseline and six months of follow up, we found some increased UAE rates in SLE patients at 12 and 18 months $(\mathrm{p}<0.05)$ when compared with healthy controls. Consistent with these findings, we also found a significant intragroup difference in the UAE rate of SLE patients throughout the study period (Friedman two way analysis of variance, $\mathrm{p}=0.01)$. As expected by the data mentioned above, the coefficients of variation for the UAE, calculated from the three overnight urine samples at each follow up visit, were larger in the SLE patients (mean 65\%, range: $26-141 \%$ ) than in the healthy controls (mean $41 \%$, range: $28-54 \%$ ). We failed to find any relation between disease activity and UAE.

Only five SLE patients had UAE values above $20 \mu \mathrm{g} / \mathrm{min}$ at some point during the study. Four of these patients had microalbuminuria $(20-200 \mu \mathrm{g} / \mathrm{min})$ only at some visits, but all were transient and of low magnitude, ranging from 20 to $41 \mu \mathrm{g} / \mathrm{min}$, and no urine sample exceeded the upper limit of the range defined as microalbuminuria ( $>200 \mu \mathrm{g} / \mathrm{min}$ ). Two of these four patients were taking a non-steroidal anti-inflammatory drug (NSAID) when microalbuminuria appeared. None of these four patients had hypertension or any data suggestive of clinical nephropathy during an additional three years of follow up after the end of the study. The fifth patient, whose UAE at baseline $(4.67 \mu \mathrm{g} / \mathrm{min})$ and six month $(4.73 \mu \mathrm{g} / \mathrm{min})$ visits were normal, presented with a nephrotic syndrome at 12 months after starting the study, and her kidney biopsy showed diffuse proliferative lupus glomerulonephritis. She was treated with prednisone and monthly doses of intravenous cyclophosphamide with complete disappearance of proteinuria (Albustix negative). Six months after the start of her treatment the UAE had decreased to normal values (4.65 $\mu \mathrm{g} / \mathrm{min}$ ). She was normotensive and had a normal renal function.

\section{Discussion}

Our results show that the group of female patients with SLE that we have studied have wide variations in the UAE, some of them reaching on occasions the microalbuminuria level. These variations were not only seen between patients and healthy controls, but also in the same patient during the 18 month follow up study with six monthly determinations. By contrast, the UAE rate from our healthy controls and their coefficients of variation (28$54 \%$ ) did not differ from the data published by other authors. ${ }^{319}$
Screening for microalbuminuria can be done by several procedures with similar results: 24 hour urine collection, overnight collection, short-term collection in the clinic, and urine albumin-creatinine ratios in random or early morning (first voided) urine samples. ${ }^{3}$ Althought a timed UAE rate, either a 24 hour or overnight collection, is the most sensitive of assays, there is a trend to use for screening purposes the first voided morning urine sample because of its simplicity. ${ }^{20}$ We have used the mean value of three, eight hour overnight urine samples collected on consecutive days as an estimation of the UAE rate, which has a good correlation with the 24 hour collection. ${ }^{21}$ Only five of 22 SLE patients without renal disease studied showed abnormal UAE in some of the four measurements. In four of these patients the increases in UAE were small and in three of them were recorded on either the first or second measurement, but not on the last occasion, suggesting that the increases were transient. During an additional follow up of three years, after the completion of this study, none of these patients has developed any renal abnormality, hypertension or diabetes. It is difficult to interpret these transient increases, but they could reflect some mesangial nephropathy, mild changes in the activity of SLE, or perhaps the use of NSAID - as it has been suggested to explain similar oscillations in UAE occurring in rheumatoid arthritis ${ }^{9}$-but when microalbuminuria developed only two patients were taking NSAIDs whereas the other two had not taken these drugs at least during the previous month. In a study reported previously mild increases in UAE in SLE patients became normal after corticosteroid treatment. ${ }^{14}$ In a fifth patient, who presented normal measurements at baseline (4.67 $\mu \mathrm{g} / \mathrm{min})$ and six month $(4.73 \mu \mathrm{g} / \mathrm{min})$ visits, a nephrotic syndrome appeared at 12 months, and a renal biopsy showed a diffuse proliferative glomerulonephritis. She was treated with prednisone and monthly doses of intravenous cyclophosphamide with complete disappearance of proteinuria (Albustix negative). Six months after the start of this treatment her UAE had returned to normal values $(4.65 \mu \mathrm{g} / \mathrm{min})$, her renal function was normal, and she was normotensive.

It seems that some patients with lupus and without clinical renal disease have wider oscillations in UAE than healthy people, although this does not seem to warrant any specific action. It is of special interest that in one of the patients, the first two UAE measurements failed to predict the development of severe renal disease. Furthermore, after treatment, her UAE returned to normal baseline values, below the level of microalbuminuria. These data are in contrast with the high predictive value of the appearance of urinary cellular casts for renal relapse in SLE patients with confirmed renal disease. ${ }^{22}$ Our findings cannot exclude that a more frequent measurement of UAE, for example at two monthly intervals, could show a rising value before proteinuria becomes obvious. The rate at which proteinuria develops in patients whose 
glomeruli are becoming inflamed is not well known, but probably in those patients who suffer from an acute inflammatory process it develops over a short period of time and cannot be predicted through UAE. Whether UAE may help to evaluate the residual glomerular damage after successful treatment of lupus glomerulonephritis merits some consideration.

1 Shihabi ZK, Konen JC, O'Connor ML. Albuminuria vs urinary total protein for detecting chronic renal disorders. nary total protein for detectir
Clin Chem 1991;37:621-4.

2 Mogensen CE, Chachati A, Christensen CK, et al. Microalbuminuria: an early marker of renal involvement in diabetes. Uremia Investigation 1986;9:85-95.

3 Mogensen CE. Microalbuminuria as a predictor of clinical diabetic nephropathy. Kidney Int 1987;31:673-89.

4 Bigazzi R, Vianchi S, Campese VM, Baldari G. Prevalence of microalbuminuria in a large population of patients with mild to moderate essential hypertension. Nephron 1992; 61:94-7.

5 Mogensen CE. Microalbuminuria predicts clinical proteinuria and early mortality in maturity-onset diabetes mellitus. N Engl Med J 1984;310:356-60.

6 Yudkin JS, Forrest RD, Jackson CA. Microalbuminuria as predictor of vascular disease in non-diabetic subjects. Lanpredictor of vascular
cet 1988 ;ii:530-3.

7 Stehouwer CDA, Nauta JJP, Zeldenrust GC, Hackeng WHL, Donker AJM, den Ottolander GJH. Urinary albumin excretion, cardiovascular disease, and endothelial albumin excretion, cardiovascular disease, and endothelial
dysfunction in non-insulin-dependent diabetes mellitus. dysfunction in non-insulin-c

Lancet 1992;340:319-23.
8 Salli L, Scalici S, Corrao S, Curiale B, Salerno L. Microproteinuria as an index of initial renal lesion in patients with rheumatoid arthritis. Clin Exp Rheumatol 1990;8:397400.

9 Pedersen LM, Nordin H, Svensson B, Bliddal H. Microalbuminuria in patients with rheumatoid arthritis. Ann Rheum Dis 1995;54:189-92.

10 Dawnay A, Wilson AGT Mc, Lamb E, Kirby JDT, Catell WR. Microalbuminuria in systemic sclerosis. Ann Rheum Dis $1992 ; 51: 384-8$.
11 Montaña E, Nolla J M, Gómez N, Roig Escofet D, Soler J. Microalbuminuria is associated with limited joint mobility in type I diabetes mellitus. Ann Rheum Dis 1995; 54:582-6.

2 Parving HH, Sorensen SF, Mogensen CE, Helin P. Urinary albumin and $\beta_{2}$-microglobulin excretion rates in patients with systemic lupus erythematosus. Scand J Rheumatol 1980;9:49-51.

13 Yamada A, Miyakawa Y, Shibata S, Kosaka K. Radioimmunoassay of urine albumin in subclinical lupus nephritis [Letter]. N Engl J Med 1980;303:643.

14 Terai C, Nojima Y, Takano K, Yamada A, Takaku F. Determination of urinary albumin excretion by radioimmunoassay in patients with subclinical lupus nephritis. Clin Nephrol 1987;27:79-83.

15 Cottiero RA, Madaio MP, Levey AS. Glomerular filtration rate and urinary albumin excretion rate in systemic lupus erythematosus. Nephron 1995;69:140-6.

16 Tan EM, Cohen AS, Fries JF, Masi AT, McShane DJ, Rothfield NF, et al. The 1982 revised criteria for the classification of systemic lupus erythematosus. Arthritis Rheum tion of systemic

17 Yeung CK, Wong KL, Wong WS, Chan KH. $\beta_{2}-$ microglobulin and systemic lupus erythematosus. J Rheumatol 1986;13:1053-8.

18 Joint National Committee. 1988 Report of the JNC on detection, evaluation and treatment of high blood pressure. Arch Intern Med 1988;148:1023-38

19 Howey JEA, Browning MCK, Fraser CG. Selecting the optimum specimen for assessing slight albuminuria, and a strategy for clinical investigation: novel uses of data on biological variation. Clin Chem 1987;33:2034-8.

20 Bennett PH, Haffner S, Kasiske BL, Keane WF, Mogensen $\mathrm{CE}$, Parving $\mathrm{HH}$, et al. Screening and management of microalbuminuria in patients with diabetes mellitus: Recommendations to the scientific advisory board of the National Kidney Foundation from and ad hoc Committee National Kidney Foundation from and ad hoc Committee ney Foundation. Am J Kidney Dis 1995;25:107-12.

21 Ellis D, Coonrod BA, Dorman JS, Kelsey SF, Becker DJ, Avner ED, et al. Choice of urine sample predictive of microalbuminuria in patients with insulin-dependent diabetes mellitus. Am J Kidney Dis 1989;13: 321-8.

22 Hebert LA, Dillon JJ, Middendorf DF, Lewis EJ, Peter JB. Relationship between appearance of urinary red blood cell/white blood cell casts and the onset of renal relapse in systemic lupus erythematosus. Am J Kidney Dis 1995; 26:432-8. 